\title{
Factors associated with recruitment to randomised controlled trials in general practice: protocol for a systematic review
}

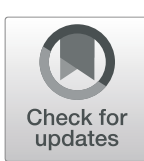

\author{
Keith R. Moffat ${ }^{1 *} \mathbb{D}$, Paul Cannon ${ }^{2} \mathbb{B}$, Wen Shi ${ }^{1}$ and Frank Sullivan ${ }^{1}$
}

\begin{abstract}
Background: Randomised controlled trials (RCTs) are frequently unable to recruit sufficient numbers of participants. This affects the trial's ability to answer the proposed research question, wastes resources and can be unethical. RCTs within a general practice setting are increasingly common and similarly face recruitment challenges. The aim of the proposed review is to identify factors that are associated with recruitment rates to RCTs in a general practice setting. These results will be used in further research to predict recruitment to RCTs.

Methods/design: The electronic databases Medline, EMBASE, Cochrane Database of Systematic Reviews, NTIS and OpenGrey will be searched for relevant articles with no limit on the date of publication. BMC Trials will be manually searched for the past 5 years. Both quantitative and qualitative studies will be included if they have studied recruitment within a general practice RCT. Only English language publications will be included. Screening, quality assessment and data extraction will be conducted by two review authors not blinded to study characteristics. Disagreement will be resolved by discussion and the involvement of a third review author if required. A narrative synthesis of the studies included will be performed.
\end{abstract}

Discussion: The review will, for the first time, systematically synthesise existing research on factors associated with recruitment rates to RCTs in general practice. By identifying research gaps to be prioritised in further research, it will be of interest to academics. It will also be of value to clinical trialists who are involved in the complex task of improving trial recruitment. Our team will use the findings to inform a prediction model of trial recruitment using machine learning.

Systematic review registration: PROSPERO, CRD42018100695. Registered on 03 July 2018.

Keywords: recruitment, general practice, randomised controlled trial

\section{Background}

Randomised controlled trials (RCTs) require a sufficient number of participants to be adequately powered. This is necessary for the trial to answer the particular research question. The significant difficulties in recruiting sufficient numbers of participants have been well established [1-3]. A review of all 151 RCTs funded by the Health Technology Assessment Programme of the UK National Institute for Health Research between 2004 and 2016 showed that only $40 \%$ of RCTs were able to recruit $100 \%$ of their

\footnotetext{
* Correspondence: krm9@st-andrews.ac.uk

'Population and Behavioural Science Division, School of Medicine, Medical \& Biological Sciences, North Haugh, St Andrews, UK

Full list of author information is available at the end of the article
}

original target, $63 \%$ were able to recruit $80 \%$ of their original target and around one third of RCTs extended their recruitment period to increase recruitment [1]. Whilst this review does not report actual recruitment as a percentage of the target sample for those studies in general practice, other studies have similarly described difficulties in recruiting individuals to general practice studies [4-6]. As a result of the challenges in recruiting participants, RCT recruitment has been set as a priority research area [7].

This systematic review will focus on recruitment rates to RCTs in general practice. The reason for this is that for many nations, care delivered in the community is the intended paradigm of health care [8,9]. Given that health care will increasingly be delivered in the community, we

(c) The Author(s). 2019 Open Access This article is distributed under the terms of the Creative Commons Attribution 4.0 International License (http://creativecommons.org/licenses/by/4.0/), which permits unrestricted use, distribution, and 
are interested in specifically researching RCTs in a general practice setting. Research findings from RCTs that have studied only a hospital setting cannot be assumed to translate to a community setting where most care is delivered. Therefore, it is important that we have a robust evidence base of treatment effectiveness for people in the community. This requires research into interventions that are specifically delivered in the community and, as such, a research priority is recruitment in a general practice setting. This has been recognised by the development of practice-based research networks internationally [10-12].

A relevant area for our own research is that of predictive modelling of recruitment to trials and the call for better models in predicting recruitment [13]. This approach is supported by a recent priority setting exercise by the James Lind Alliance (PRioRiTy) for research into RCT recruitment. A major priority identified for research into RCT recruitment included: "What are the best ways to predict recruitment rates to a randomized trial and what impact do such predictions have on recruitment?" [14].

We aim to develop a predictive model of RCT recruitment rates using machine-learning methods. So that the appropriate data are used for this model, it is necessary to identify the factors that have hitherto been identified as being associated with recruitment to RCTs. Although this will be of broader interest, we will be using the outcomes from this systematic review to select appropriate datasets for our model. It is important to state that the factors identified in the systematic review need not have been shown to predict or affect recruitment, since machine-learning algorithms will determine predictability during development.

Other systematic reviews in this research area include the Cochrane reviews by Treweek et al., which have focused upon interventions to improve recruitment to RCTs [15-17]. Whilst this is clearly important, for our purposes this would not be sufficient because they identify factors associated with recruitment in the context of an intervention. For example, there are studies that retrospectively investigate reasons for poor recruitment, which is of value for our purpose but is not interventional by type [18]. Additionally, we expect there will be interventional studies aiming to improve recruitment rates but that are not randomised or quasi-randomised, which would have been omitted by Treweek et al.'s selection criteria. An ongoing Cochrane qualitative evidence synthesis of factors that impact recruitment to randomised trials [19] is similarly not broad enough for our purposes, since it will be including only studies investigating the participant perspective in recruitment. As such, it will not investigate the perspective of the recruiter (personal correspondence, 12 January 2018), e.g. general practices as a unit or general practitioners. This study will include both recruiters and participants.
Furthermore, none of these reviews is focused on the area of general practice, as they look at recruitment more widely.

\section{Methods/design}

The protocol has been developed according to the PRISMA guidelines for systematic review protocols (PRISMA-P) [20]. The completed PRISMA-P checklist for this protocol is given as Additional file 1. The protocol has been registered with PROSPERO (registration number CRD42018100695), which can be accessed via the following webpage: https://www.crd.york.ac.uk/PROSPERO/display_record.php?RecordID $=100695$

Aim

The aim of this systematic review is to identify factors associated with recruitment of individuals and practices to RCTs in general practice.

\section{Eligibility criteria}

The following criteria will be used in selecting studies for the systematic review:

1. Study design

Any primary study design that investigates recruitment to RCTs in general practice will be included. RCTs based in general practice will not themselves be included unless they have also investigated recruitment. Qualitative and quantitative studies will be included.

2. Participants

Studies of any types of participant will be included. Studies will be included both where the focus is on recruitment of practices to RCTs as well as individual participants.

3. Interventions Studies of any types of intervention that have targeted recruitment will be included.

4. Comparators

Studies of any types of comparator will be included. Interventional study designs are likely to compare to the usual recruitment method. Observational studies may report variations based on sociodemographic variables or system differences, e.g. research networks and payments.

5. Outcomes

Only studies where at least one outcome has focused on recruitment will be included. Examples of such outcomes include the number of participants recruited, percentage of recruitment target achieved and time to first participant recruited. The different recruitment outcomes will be collated and described.

6. Setting 
Only RCTs investigating recruitment within a general practice setting will be included. General practices can be involved in recruitment in two main ways. The first is as the locus of the intervention such that recruited participants are randomised to an intervention based in the practice. The second is where the practice is used to recruit participants to a trial that is not based within a general practice setting. Only studies in the former setting will be included.

7. Language

Only studies in English will be included.

\section{Information sources}

The following databases were searched for relevant studies:

- Ovid MEDLINE(R) In-Process \& Other NonIndexed Citations and Ovid MEDLINE, 1946 to present via OvidSP

- Embase 1947-Present, updated daily via OvidSP

- Cochrane Central Register of Controlled Trials (CENTRAL): Issue 4 of 12, April 2018 \& Cochrane Database of Systematic Reviews (CDSR): Issue 5 of 12, May 2018 via the Cochrane Library

All databases were searched on 23 May 2018. In addition, the websites OpenGrey (http://www.opengrey.eu/) and the National Technical Reports Library (https://ntrl.ntis.gov/ NTRL/) were searched on 1 June 2018. The journal BMC Trials will be manually searched for the preceding 5 years (from the 1st of June 2013 to the 1st of June 2018). Experts in the field of trial recruitment were asked to suggest further important articles for inclusion.

\section{Search strategy}

The search strategy was supported by a health information specialist with systematic review experience (PC). The search strategy used both text words and relevant indexing for controlled or multicentre trials, selection and recruitment, and general practices and practitioners. Citation and bibliographic searches will be conducted on all included studies to identify additional relevant studies. The search will be updated towards the end of the review. The full search strategy is given in Additional file 2.

\section{Study records}

Literature search results will be exported to the systematic review software DistillerSR. Based on the inclusion and exclusion criteria, the review team will develop screening questions and forms, which will be tested by a calibration exercise prior to implementation. These will be uploaded to DistillerSR along with citation abstracts and full articles. All members of the review team not familiar with DistillerSR or the subject area will receive training. A PRISMA diagram [21] will be completed to show the numbers of studies selected at different levels of assessment.

\section{Selection process}

Titles and abstracts of studies found using the search strategy and from other sources will be independently screened by two reviewers (KM and WS). The full text of those studies that potentially meet the inclusion criteria will be retrieved and independently assessed for eligibility by two reviewers. Any disagreements over the eligibility of studies will be resolved through discussion with a third reviewer (FS). Reasons for excluding studies will be recorded. The reviewers will not be blinded to the journal titles, study authors or institutions.

\section{Data collection process}

Standardised forms will be used to extract data into DistillerSR. Two review authors (KM and WS) will extract data independently from the studies included, and conflicts will be resolved through discussion. Any disagreements will be resolved with a third reviewer (FS). Extracted data will include demographic information, methodology and outcomes as well as measured recruitment metrics. Study authors will be contacted if any relevant data are incomplete.

\section{Outcomes}

The outcome will be study recruitment. There are many ways of measuring this, including numbers, proportions and rates. These will be captured during data extraction. Additionally, recruitment outcomes of both practices and individuals will be extracted.

\section{Risk of bias}

Two reviewers will independently assess the risk of bias. This will be assessed at the study level. The Cochrane collaboration tool for assessing the risk of bias [22] will be used for RCTs and the relevant CASP checklist will be used for non-RCT studies [23]. This information will be tabulated along with the corresponding studies and will inform our discussion of the quality of the evidence base on this topic.

\section{Data synthesis}

A narrative synthesis of the included studies will be performed. The information will be presented in text and tabular format to summarise the characteristics and results of the included studies as well as to explain the outcomes within and between the studies. Guidance from the Centre for Reviews and Dissemination will inform the synthesis [24]. It is anticipated that included studies will have a high risk of bias due to their design. For this reason, all studies will be included in the synthesis with an appropriate discussion of their limitations. 


\section{Discussion}

This will be the first time that a systematic review has synthesised factors associated with recruitment to RCTs in general practice. In doing so, it will comprehensively identify recruitment factors that should be considered by both those conducting trials and also trial researchers. The quality of the included studies will inform further research in trial recruitment by identifying research gaps reflected by studies of poorer quality. Finally, the findings will directly inform a model that our team is developing to predict recruitment to RCTs in general practice using machine learning methods.

\section{Additional files}

Additional file 1: PRISMA-P 2015 checklist. (DOCX 32 kb)

Additional file 2: Search strategy (PDF $212 \mathrm{~kb}$ )

\section{Acknowledgements}

Not applicable.

\section{Funding}

This study is supported by a PhD studentship provided by the University of St Andrews. The university had no role in the development of the protocol.

\section{Availability of data and materials}

Not applicable.

\section{Authors' contributions}

KRM conceived of the study, drafted the protocol and contributed to the design and search strategy. PC contributed to development of the search strategy and running the search. FM is supervisor for the study and contributed to the design of the study. All authors read and approved the final manuscript.

\section{Ethics approval and consent to participate}

Not applicable.

\section{Consent for publication}

Not applicable.

\section{Competing interests}

The authors declare that they have no competing interests.

\section{Publisher's Note}

Springer Nature remains neutral with regard to jurisdictional claims in published maps and institutional affiliations.

\section{Author details}

1Population and Behavioural Science Division, School of Medicine, Medical \& Biological Sciences, North Haugh, St Andrews, UK. ${ }^{2}$ Information Services, University of Glasgow, Hillhead Street, Glasgow G12 8QE, UK.

Received: 9 August 2018 Accepted: 3 April 2019

Published online: 10 May 2019

\section{References}

1. Walters SJ, Bonacho dos Anjos Henriques-Cadby I, Bortolami O, Flight L, Hind D, Jacques RM, Knox C, Nadin B, Rothwell J, Surtees M, et al. Recruitment and retention of participants in randomised controlled trials: a review of trials funded and published by the United Kingdom Health Technology Assessment Programme. BMJ Open. 2017;7:e015276.

2. McDonald AM, Knight RC, Campbell MK, Entwistle VA, Grant AM, Cook JA, Elbourne DR, Francis D, Garcia J, Roberts I, et al. What influences recruitment to randomised controlled trials? A review of trials funded by two UK funding agencies. Trials. 2006;7:9.
3. Sully $B G$, Julious $S A$, Nicholl J. A reinvestigation of recruitment to randomised, controlled, multicenter trials: a review of trials funded by two UK funding agencies. Trials. 2013;14:166

4. van der Gaag WH, van den Berg R, Koes BW, Bohnen AM, Hazen LM, Peul WC, Voogt L, Verhagen AP, Bierma-Zeinstra SM, Luijsterburg PA. Discontinuation of a randomised controlled trial in general practice due to unsuccessful patient recruitment. Br J Gen Pract Open 2017;1(3): bjgpopen17X101085.

5. Schreijenberg M, Luijsterburg PAJ, Van Trier YDM, Rizopoulos D, Koopmanschap MA, Voogt L, Maher CG, Koes BW. Discontinuation of the PACE Plus trial: problems in patient recruitment in general practice. BMC Musculoskelet Disord. 2018;19(1):146.

6. Foster JM, Sawyer SM, Smith L, Reddel HK, Usherwood T. Barriers and facilitators to patient recruitment to a cluster randomized controlled trial in primary care: lessons for future trials. BMC Med Res Methodol. 2015;15:18.

7. Tudur Smith C, Hickey H, Clarke M, Blazeby J, Williamson P. The trials methodological research agenda: results from a priority setting exercise. Trials. 2014;15:32.

8. Primary Care. http://www.gov.scot/Topics/Health/Services/Primary-Care. Accessed 18 Feb 2018.

9. The World Health Report 2008 - primary Health Care (Now More Than Ever). Geneva: World Health Organisation; 2008.

10. Sullivan F, Hinds A, Pitkethly M, Treweek S, Wilson P, Wyke S. Primary care research network progress in Scotland. Eur J Gen Pract. 2014:20(4):337-42.

11. Rørtveit $G$. Research networks in primary care: an answer to the call for better clinical research. Scand J Prim Health Care. 2014;32(3):107-9.

12. Mold JW. Primary care research conducted in networks: getting down to business. J Am Board Family Med. 2012;25(5):553-6.

13. Barnard KD, Dent $L$, Cook A. A systematic review of models to predict recruitment to multicentre clinical trials. BMC Med Res Methodol. 2010;10:63.

14. Healy P, Galvin S, Williamson PR, Treweek S, Whiting C, Maeso B, Bray C, Brocklehurst $\mathrm{P}$, Moloney MC, Douiri A, et al. Identifying trial recruitment uncertainties using a James Lind Alliance Priority Setting Partnership - the PRioRiTy (Prioritising Recruitment in Randomised Trials) study. Trials. 2018; 19(1):147.

15. Treweek S, Pitkethly M, Cook J, Kjeldstrom M, Taskila T, Johansen M, Sullivan F, Wilson S, Jackson C, Jones $\mathrm{R}$, et al. Strategies to improve recruitment to randomised controlled trials. Cochrane Database Syst Rev. 2010;4.

16. Treweek $S$, Lockhart $P$, Pitkethly $M$, Cook JA, Kjeldstrom M, Johansen $M$, Taskila TK, Sullivan FM, Wilson S, Jackson C, et al. Methods to improve recruitment to randomised controlled trials: Cochrane systematic review and meta-analysis. BMJ Open. 2013;3(2):e002360.

17. Treweek S, Pitkethly M, Cook J, Fraser C, Mitchell E, Sullivan F, Jackson C, Taskila TK, Gardner H. Strategies to improve recruitment to randomised trials. Cochrane Database Syst Rev. 2018;2.

18. Bell-Syer SE, Thorpe LN, Thomas K, Macpherson H. GP Participation and Recruitment of Patients to RCTs: Lessons from Trials of Acupuncture and Exercise for Low Back Pain in Primary Care. Evid Based Complement Alternat Med. 2011;2011:687349.

19. Houghton C, Dowling M, Meskell $\mathrm{P}$, Hunter A, Gardner $\mathrm{H}$, Conway A, Treweek S, Sutcliffe K, Noyes J, Devane D, et al. Factors that impact on recruitment to randomised trials in health care: a qualitative evidence synthesis. Cochrane Database Syst Rev. 5(2017).

20. Moher D, Shamseer L, Clarke M, Ghersi D, Liberati A, Petticrew M, Shekelle P, Stewart LA. Preferred reporting items for systematic review and metaanalysis protocols (PRISMA-P) 2015 statement. Syst Rev. 2015;4(1):1.

21. Moher D, Liberati A, Tetzlaff J, Altman DG. Preferred reporting items for systematic reviews and meta-analyses: the PRISMA statement. PLoS Med. 2009:6(7):e1000097.

22. The Cochrane Collaboration's tool for assessing risk of bias. https:// handbook-5-1.cochrane.org/chapter 8/table 85 a the cochrane collaborations_tool_for_assessing.htm. Accessed 29 Apr 2019.

23. CASP Checklists. https://casp-uk.net/casp-tools-checklists/. Accessed 26 June 2018

24. Systematic Reviews: CRD's Guidance for Undertaking Reviews in Health Care. York: Centre for Reviews and Dissemination; 2009. 